\title{
Randomized controlled trials
}

\author{
David F Marks
}

Potential competing interests: The author(s) declared that no potential competing interests exist.

Randomized controlled trials (RCTs) involve the systematic comparison of interventions using a fully controlled application of one or more 'treatments' with a random allocation of participants to the different treatment groups. The statistical tests that are available have as one of their assumptions that participants have been randomly assigned to conditions. In realworld settings of clinical and health research, the so-called 'gold standard' of the RCT cannot always be achieved in practice, and in fact may not be desirable for ethical reasons. 